\title{
ABORDAJE OPORTUNO DE COLESTASIS NEONATAL. REPORTE DE CASO, HOSPITAL PEDIÁTRICO “DR. ROBERTO GILBERTELIZALDE”
}

\author{
EARLY APPROACH OF NEWBORN CHOLESTASIS IN THE "DR. ROBERTO GILBERT ELIZALDE" \\ PEDIATRIC HOSPITAL. ACASE REPORT
}

\author{
ABORDAGEM OPORTUNA DA COLESTASE NEONATAL. CASO REPORTADO NO HOSPITAL PEDIÁTRICO "DR. ROBERTO \\ GLLBERTELIZALDE
}

\author{
JIMMY PAZMIÑO ARROBA', VERÓNICA ZAPATA MATERON² \\ 1 Hospital Dr. Roberto Gilbert Elizalde, Guayaquil, Ecuador \\ 2 Universidad Católica de Santiago de Guayaquil; Guayaquil, Ecuador
}

Resumen

La atresia de vías biliares es una colangiopatía progresiva, obstructiva y obliterativa, que puede involucrar tanto las vías biliares intrahepáticas como extrahepáticas, la cual representa una de las principales causas de transplante hepático en la población pediátrica. El objetivo es dar a conocer la importancia del diagnóstico oportuno, junto al manejo multidisciplinario de la colestasis neonatal. Se presenta el caso de paciente femenino de 2 meses de edad, con cuadro clínico de 2 semanas de evolución, que presentó acolia, coluria e ictericia, que progresa a insuficiencia hepática; se realiza biopsia cuyo resultado fue atresia de vías biliares. En los días subsecuentes, la paciente evolucionó a insuficiencia hepática y shock séptico hipotensivo refractario, que finalmente la llevó al paro cardiorrespiratorio y la muerte. En el abordaje de la colestasis neonatal, el reconocimiento de los signos semiológicos, son datos claves para su sospecha clínica. Se concluye que el diagnóstico oportuno, dentro de los primeros 45 días de vida de los pacientes con atresia de vías biliares es crucial en el pronóstico del mismo y así evitar el desarrollo de la enfermedad hepática crónica e insuficiencia hepática.

PALABRAS CLAVE: atresia biliar, colestasis, diagnóstico, ictericia neonatal, insuficiencia hepática.

Abstract

Biliary atresia is a progressive, obstructive and obliterative cholangiopathy that can affect the intrahepatic as well as the extrahepatic biliar ducts. This disease represents one of the main causes of hepatic transplant in the pediatric population. For this reason, the core objective is to communicate the importance of an early diagnosis with a multidisciplinary management. We report the case of a female, 2 months patient that came with jaundice, acholia and coluria of 2-weeks that progress to hepatic failure and septic refractory hypotensive shock that finally ended up in a cardiac arrest and death. In assessment of a newborn cholestasis the semiological signs are key to a preliminary diagnosis. The early diagnosis, within the first 45 days in patients with biliary atresia is of paramount importance to the prognosis and prevention of chronic hepatic disease and hepatic failure.

KEYWORDS: biliary atresia, cholestasis, diagnosis, jaundice neonatal, hepatic insufficiency.

Resumo

A atresia das vias biliares é uma colangiopatia progressiva, obstrutiva e obliterativa, que pode envolver tanto os ductos biliares intra-hepáticos como extra-hepáticos, o que representa uma das principais causas de transplante hepático na população pediátrica. É por este motivo que o principal objetivo é dar a conhecer a importância do diagnóstico atempado, juntamente com a gestão multidisciplinar. A seguir, apresentamos o caso de uma paciente do sexo feminino com 2 meses de idade, com quadro clínico de 2 semanas de evolução, apresentando acolia, colúria e icterícia, evoluindo para insuficiência hepática, na qual foi realizada biópsia, que resultou em atresia da via biliar. Nos dias seguintes, o paciente evoluiu com insuficiência hepática e choque séptico hipotensivo refratário, o que levou à parada cardiorrespiratória e à morte. Na abordagem da colestase neonatal, o reconhecimento de sinais semiológicos são dados fundamentais para a suspeita clínica. Conclui-se que o diagnóstico oportuno, dentro dos primeiros 45 dias de vida dos pacientes com atresia biliar, é crucial no prognóstico dos mesmos e, assim, prevenir o desenvolvimento de doença hepática crônica e até insuficiência hepática.

PALAVRAS-CHAVE: atresia biliar, colestase, diagnóstico, icterícia neonatal, insuficiência hepática. 
INTRODUCCIÓN

El término "colestasis" define a la alteración del flujo biliar, con retención de bilirrubina, ácidos biliares y otros componentes de la bilis. ${ }^{1}$ El diagnóstico diferencial de las distintas entidades causantes de ictericia neonatal puede ser difícil; sin embargo, en el contexto del cuadro clínico característico de un neonato que desarrolla la triada clásica de ictericia con acolia y coluria, de inicio entre las 2 a 6 semanas de vida, constituyen una alta sospecha de atresia de vías biliares. ${ }^{2}$

En el contexto epidemiológico, del 60 al 80\% de los recién nacidos pueden desarrollar ictericia; y de estos, entre 1:2500 a 1:5000 se relaciona a colestasis neonatal. ${ }^{3-5}$

La atresia de vías biliares se define como una colangiopatía progresiva, obstructiva y obliterativa tanto de las vías biliares intrahepáticas como extrahepáticas. En la evolución natural de esta enfermedad, a partir de los 2-3 meses, van apareciendo signos de hipertensión portal, y si no se trata, la colestasis resultante conduce a cirrosis e insuficiencia hepática. ${ }^{6,7}$ La portoenterostomía (Kasai) realizada de forma temprana ha impactado significativamente en la sobrevida de los pacientes con atresia de vías biliares; 8 por lo que es necesario que el personal de salud utilice herramientas prácticas, como lo constituye aplicar el sistema de esquema de identificación en escala de colores de las heces del neonato.

\section{CASO CLÍNICO}

Lactante de 2 meses de edad, con antecedente de un hermano fallecido a los 6 meses de vida por hepatopatía. Ingresa al área de hospitalización por cuadro clínico de 15 días de evolución caracterizado por ictericia generalizada y edema de miembros inferiores relacionado con desnutrición grave (percentil $<3$ de índice de masa corporal). A su ingreso con suficiencia cardiorrespiratoria; sin embargo, llama la atención la presencia de coluria y acolia, por lo que se inició estudio de colestasis. En el interrogatorio, madre refiere que tanto la orina como las deposiciones han tenido similares características hace más de un mes.

Los resultados de laboratorio, reportaron hiperbilirrubinemia a predominio de bilirrubina directa, elevación de transaminasas, anemia, hipoalbuminemia y tiempos de coagulación prolongados.
En la ecografía de abdomen se observó imagen anecoica compatible con vesícula hipoplásica (Figura 1). Por la alta sospecha de atresia de vías biliares se solicitó una colangiorresonancia, que reportó hígado aumentado de tamaño, ausencia de vías biliares extrahepáticas con vesícula biliar hipoplásica, (Figura 2) y finalmente por biopsia hepática se encontró atresia de vías biliares intrahepáticas con esteatosis, completando así el diagnóstico de atresia de vías biliares intra y extrahepáticas. En los días subsecuentes, el paciente evolucionó a insuficiencia hepática y shock séptico hipotensivo refractario, que finalmente lo llevó al paro cardiorrespiratorio y la muerte.

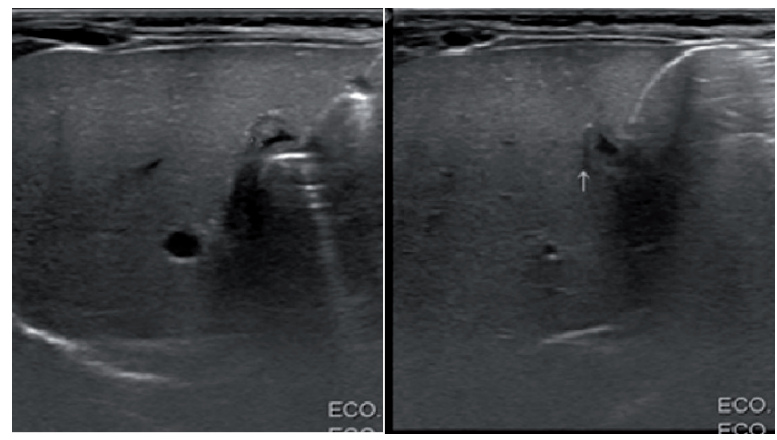

Figura 1. Ecografía de abdomen: imagen anecoica de paredes gruesas que se proyecta en topografía de vesícula.

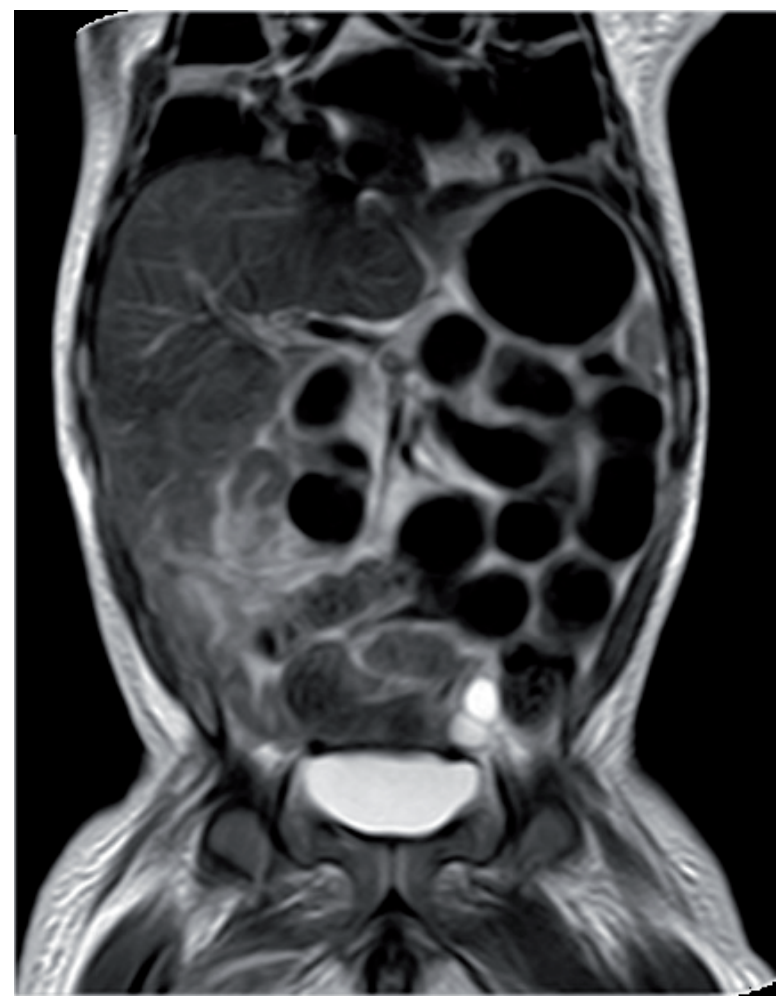

figura 2. Colangiorresonancia: se visualiza vesícula hipoplástica, no se visualizan vías biliares extrehepáticas 


\section{DISCUSIÓN}

La edad del diagnóstico está fuertemente correlacionada con la supervivencia de los pacientes, puesto que de este tiempo depende la primera intervención quirúrgica a realizar, la portoenterostomía de Kasai (PK), evita el daño rápidamente progresivo a nivel hepático. Los mejores resultados se obtienen cuando la PK se realiza antes de los 45 días de vida. ${ }^{9}$ Esta técnica quirúrgica, consiste en la anastomosis términolateral de un asa yeyunal en Y Roux a la placa ductal tras la búsqueda de conductos intrahepáticos permeables en el hilio; el objetivo es el restablecimiento del flujo biliar. Tras la cirugía, un $30 \%$ no mejorará el flujo biliar y otro $30 \%$ lo harán de forma parcial, precisando ambos grupos un trasplante hepático en los meses siguientes. ${ }^{10}$

Muchos son los factores para el diagnóstico tardío de estos pacientes, puesto que no es común la revisión de las heces de los neonatos o preguntar acerca de las características de las mismas; las causas de ictericia en la etapa neonatal son múltiples por lo que el diagnóstico de atresia de vías biliares no encabeza la lista de las posibilidades; las visitas del niño sano están estandarizadas de manera mensual, lo que conlleva al retraso del diagnóstico hasta por 30 días según la siguiente visita programada por el pediatra. En ciertas escuelas, como la canadiense, se recomienda la cuantificación de bilirrubina total y conjugada en todo neonato con ictericia después del día 15 de vida, caracterizando a la colestasis por la elevación de la bilirrubina directa sérica mayor a $1 \mathrm{mg} / \mathrm{dl}$ (si la bilirrubina total es menor a $5 \mathrm{mg} / \mathrm{dl}$ ) o más del $20 \%$ de la bilirrubina total. ${ }^{11}$

Según un estudio piloto realizado en Taiwan para detectar el color de las heces en los neonatos según la cartilla de colores (figura 3) en la consulta médica de control, se logró el diagnóstico precoz de pacientes con colestasis, la sensibilidad, especificidad y el valor predictivo positivo de la aplicabilidad del método de verificación del color de las heces de los neonatos, fueron del $89.7 \%$, $99.9 \%$ y $28.6 \%$, respectivamente. ${ }^{12}$ Todos los lactantes detectados, fueron operados antes de los 90 días de vida, y la tasa de supervivencia a los 5 años aumentó de 37 a 64\%,9 cambiando el pronóstico de vida de los pacientes. ${ }^{13}$ De esta manera se han desarrollado estrategias para la detección temprana de esta enfermedad logrando la conformación de un equipo integrado por padres y pediatras.

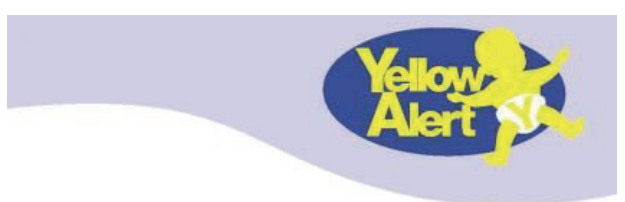

One of these babies has liver disease. Can you tell which one?

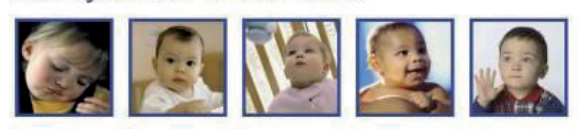

Difficult to decide? What can you do? Check your baby's urine colour
If yellow - tell your Doctor, health visitor or midwife Check your baby's stool colour:

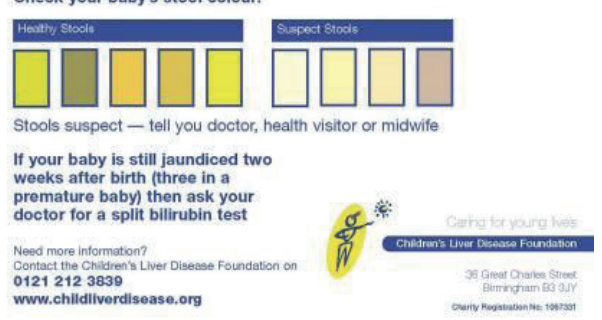

Figura 3. Cartilla yellow alert. Fuente: www.yellowalert.org

\section{CONCLUSIONES}

La atresia de vías biliares, es la causa más habitual de colestasis crónica en la infancia y la indicación más frecuente de trasplante hepático en niños. El diagnóstico precoz seguido del tratamiento quirúrgico en las primeras semanas de vida, mejora sustancialmente el pronóstico de la enfermedad y la calidad de vida. ${ }^{14}$ La ictericia, la acolia y la coluria es la triada de signos clave para su detección temprana en los 3 primeros meses de vida, y así evitar el desarrollo de la enfermedad hepática crónica e inclusive insuficiencia hepática. ${ }^{15}$

El tiempo del diagnóstico es crucial para el pronóstico del paciente. Se ha creado una estrategia de tamizaje basada en una cartilla con el color normal y patológico de las heces. ${ }^{16} \mathrm{El}$ empleo de dicha cartilla es un método simple, eficiente y aplicable de detección, mejorando radicalmente la supervivencia de estos pacientes, y evitando la muerte prematura.

\section{REFERENCIAS BIBLIOGRÁFICAS}

1. D’Amato M, Ruiz P, Aguirre K, Gómez S. Colestasis en pediatría. Rev Col Gastroenterol [Internet]. 2016;31(4):409-17. Disponible en: http://www.scielo. org.co/pdf/rcg/v31n4/v31n4a11.pdf

2. Ramonet M. Colestasis neonatal. En: Programa Nacional de Actualización Pediátrica 2015. Buenos Aires: Sociedad Argentina de Pediatría; 2015. p. 43-72. 
3. Chiu C, Chen P, Chan C, Chang M, Wu T. Biliary atresia in preterm infants in Taiwan: A Nationwide Survey. The Journal of Pediatrics.2013; 163(1):100103.e1.

4. Chardot C, Buet C, Serinet M, Golmard J, Lachaux A, Roquelaure B et al. Improving outcomes of biliary atresia: French national series 1986-2009. Journal of Hepatology. 2013;58(6):1209-1217.

5. Frauca Remacha E, Muñoz Bartolo G. Colestasis en el lactante. Asociación Española Pediátrica; Sociedad Española Gastroenterología, Hepatología y Nutrición Pediátrica [Internet]. 2010;2a edición:187. Disponible en: https://www.aeped.es/sites/default/files/documentos/colestasis.pdf

6. Feldman AG, Sokol RJ. Neonatal Cholestasis. NeoReviews. 2013;14(2):e63-73.

7. Davenport M, Tizzard SA, Underhill J, Mieli-Vergani G, Portmann B, Hadžić N. The biliary atresia splenic malformation syndrome: A 28-year single-center retrospective study. J Pediatr. 2006;149(3):393-400

8. Chen S M, Chang M H, Du J C. et al Screening for biliary atresia by infant stool colour card in Taiwan. Pediatrics 2006. 1171147-1154.

9. NASPGHAN. Guideline for the Evaluation of Choles tatic Jaundice in Infants: Recommendations of the North American Society for PediatricGastroenterology, Hepatology and Nutrition. J Pediatr Gastroenterol Nutr 2004;39(2):115-128.
10. Dayan B, Ledder O, Verstandig AG, Fisher D, Cytter-Kuint R, Granot E. Bile lake post Kasai hepatoportoenterostomy in biliary atresia. Harefuah. 2015;154(3):171-4.

11. Schreiber, R. A., \& Butler, A. Screening for biliary atresia: it's in the cards. Canadian Family Physician. 2017; 63(6), 424-425.

12. Chen, S. M., Chang, M. H., Du, J. C., Lin, C. C., Chen, A. C., Lee, H. C., ... \& Lai, M. W. Screening for biliary atresia by infant stool color card in Taiwan. Pediatrics. 2006; 117(4), 1147-1154.

13. Masucci L, Kaczorowski J, Collet J, Lutley P, Espinosa Vet al. Home-based screening for biliary atresia using infant stool colour cards: A large-scale prospective cohort study and cost-effectiveness analysis. Journal of Medical Screening. 2014;21(3):126-132.

14. Monroy-Teniza, Z. A., Flores-Calderón, J., \& VillasísKeever, M. Á. Factores pronósticos relacionados con la mortalidad de niños con atresia de vías biliares. Revista Médica del Instituto Mexicano del Seguro Social. 2015;53(3).

15. Lakshminarayanan B, Davenport M. Biliary atresia: A comprehensive review. Journal of Autoimmunity. 2016;73:1-9.

16. Ramonet MD, Gómez S, Morise S, Parga L, et al. Detección precoz de la colestasis neonatal en las heces por el mètodo de tamizaje con tarjetas colorimètricas. Arch Argent Pediatr 2013;111(2):135-9. 